\title{
Influence of Surface Interactions on Folding and Forced Unbinding of Semiflexible Chains
}

\author{
V. Barsegov ${ }^{\dagger}$ and D. Thirumalai* ${ }^{\dagger}, \dot{\hbar}$ \\ Biophysics Program, Institute for Physical Science and Technology, and Department of Chemistry and \\ Biochemistry, University of Maryland, College Park, Maryland 20742
}

Received: July 11, 2005; In Final Form: September 7, 2005

\begin{abstract}
We have investigated the folding and forced unbinding transitions of adsorbed semiflexible polymer chains using theory and simulations. These processes describe, at an elementary level, a number of biologically relevant phenomena that include adhesive interactions between proteins and tethering of receptors to cell walls. The binding interface is modeled as a solid surface, and the wormlike chain (WLC) is used for the semiflexible chain (SC). Using Langevin simulations, in the overdamped limit we examine the ordering kinetics of racquet-like and toroidal structures in the presence of an attractive interaction between the surface and the polymer chain. For a range of interactions, temperature, and the persistence length, $l_{\mathrm{p}}$, we obtained the monomer density distribution, $n(x)$, ( $x$ is the perpendicular distance of a tagged chain end from the surface) for all of the relevant morphologies. There is a single peak in $n(x)$ inside the range of attractive forces, $b$, for chains in the extended conformations, whereas in racquet and toroidal structures there is an additional peak at $x \approx$ $b$. The simulated results for $n(x)$ are in good agreement with theory. The formation of toroids on the surface appears to be a first-order transition as evidenced by the bimodal distribution in $n(x)$. The theoretical result underestimates the simulated $n(x)$ for $x \ll b$ and follows $n(x)$ closely for $x \geq b$; the calculated density agrees exactly with $n(x)$ in the range $x \ll b$. The chain-surface interaction is probed by subjecting the surface structures to a pulling force, $f$. The average extension, $\langle x(f)\rangle$, as a function of $f$ exhibits a sigmoidal profile with sharp all-or-none transition at the unfolding force threshold $f=f_{\mathrm{c}}$ which increases for more structured states. Simulated $\langle x(f)\rangle$ compare well with the theoretical predictions. The critical force, $f_{\mathrm{c}}$, is a function of $l_{\mathrm{s}} l_{\mathrm{c}}$ for a fixed temperature, where $l_{\mathrm{c}}$ and $l_{\mathrm{s}}$ are the length scales that express the strength of the intramolecular and $\mathrm{SC}-$ surface attraction, respectively. For a fixed $l_{\mathrm{s}}, f_{\mathrm{c}}$ increases as $l_{\mathrm{p}}$ decreases.
\end{abstract}

\section{Introduction}

Interactions between biomolecules and surfaces are important in a number of biological phenomena. Binding and unbinding of proteins from macromolecular complexes are involved in the regulation of biological functions. ${ }^{1-3}$ Adsorption of fibrinogen influences the adhesion of leukocytes, microphages, or platelets. In addition, interaction between proteins, DNA, and RNA are mediated by biological membranes. ${ }^{4-6}$ In the crowded cellular conditions, protein - protein and DNA - protein interactions take place in confined geometries in which surface interactions are vital. For instance, interaction between P-selectin receptors and their specific ligands is mediated by a flat and shallow binding interface. 7,8 Besides these situations, which are obviously relevant in biology, there are a number of situations in polymer science in which interactions with surfaces are important. ${ }^{9-12}$ These include nanolubrication, which involves interaction between surfaces that are mediated by polymers. Design of nanoscale materials and biologically inspired self-assembling systems also requires an understanding of how heteropolymers and biomolecules interact with surfaces. Recent advances in atomic force microscopy ${ }^{7,13,14}$ has allowed a direct probe of the energetics of interaction between adsorbed proteins with other biomolecules. ${ }^{1,15-19}$ The potential applications of polymersurface models to a number of problems has prompted us to

* Corresponding author. Phone: 301-405-4803; fax: 301-314-9404; e-mail: thirum@glue.umd.edu.

†iophysics Program, Institute for Physical Science and Technology.

$\div$ Department of Chemistry and Biochemistry. develop a theoretical approach that can be used in conjunction with AFM experiments to decipher biomolecule-surface interactions.

There have been numerous studies of the adsorption of flexible polymers adsorbed on solid surfaces that find applications in many aspects of colloidal and interface science. ${ }^{10,11,12,20}$ However, many biomolecules, including DNA, RNA, and proteins, are better described using wormlike chain (WLC) models. ${ }^{1,21,22}$ Thus, it is important to provide a theoretical description of the interactions between semiflexible chains ${ }^{22-24}$ and interfaces. The purpose of this paper is to address the following specific questions: (i) It is known that DNA, a semiflexible polymer, undergoes a coil-globule transition in the presence of osmolytes or multivalent cations. ${ }^{23,25}$ Simulations of semiflexible chains in poor solvents ${ }^{20,26}$ have been used to understand the kinetics and pathways of transitions from extended conformations to collapsed toroidal structures. The coil-globule transition in stiff chains in the bulk occurs through a series of metastable racquet structures. ${ }^{20,26}$ How does the interaction with the surface alter the morphology and kinetics of such transitions? This question is relevant even for DNA collapse in cells where the DNA compaction takes place in the presence of interactions with their large biomolecules in restricted spaces; (ii) AFM experiments are likely to provide the most direct data for the strength of interaction between semiflexible biomolecules. In these experiments, one of the molecules of interest is anchored onto the surface while force is applied to the end of the other. The unbinding force can be calculated from the force-extension profiles. These experiments 


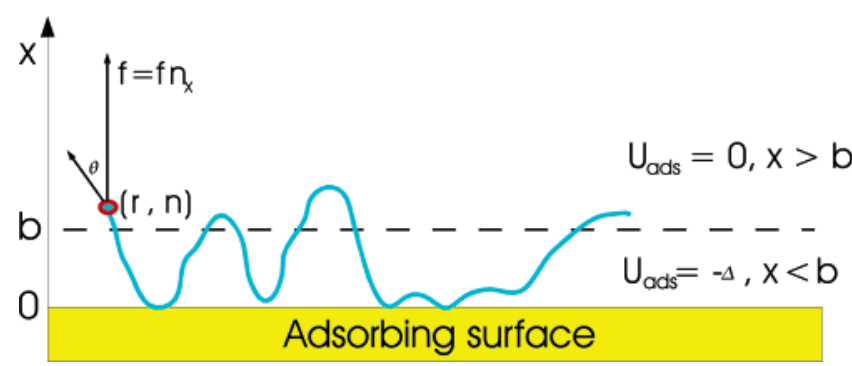

Figure 1. Schematic of a semiflexible chain (blue) adsorbed on the surface (yellow). The free end (r, n) makes an angle $\theta=\operatorname{arc}$ $\cos \left[\mathbf{n} \cdot \mathbf{n}_{x} /|\mathbf{n}| \cdot\left|\mathbf{n}_{x}\right|\right]$ with the direction $\mathbf{n}_{x}$ of the pulling force $\mathbf{f}=f \mathbf{n}_{x}$. For clarity, the chain is shown as extended, which is realized only when the SC-surface interaction is strong. The interaction between the monomers of the chain and the surface is attractive in the range $0 \leq x$ $\leq b$ where $x$ is the distance perpendicular to the surface. The strength of the interaction is $\Delta$. In the Langevin simulations, we replace the square-well potential by the Lennard-Jones potential (eq 17).

raise a question, namely, what are the adhesive forces between semiflexible polymer and a surface? We address this question using theory ${ }^{12,27,28}$ and simulations for a WLC model interacting with a solid surface.

In the absence of the surface, the morphologies of the semiflexible chain (SC) are determined by thermal fluctuations and an interplay of the chain persistence length, $l_{\mathrm{p}}$, and intramolecular condensation length $l_{\mathrm{c}}=\sqrt{l_{\mathrm{p}} k_{\mathrm{B}} T / u_{\mathrm{m}}}$ where $T$ is the temperature and $u_{\mathrm{m}}$ is the effective intramolecular attractive energy per unit length. ${ }^{20}$ In the presence of a surface another length scale $l_{\mathrm{s}}=\sqrt{l_{\mathrm{p}} k_{\mathrm{B}} T / u_{\mathrm{ads}}}$, where $u_{\mathrm{ads}}$ is the attractive SCsurface interaction energy per unit length, plays an essential role in the determination of the structures. The interplay of $l_{\mathrm{p}}$, $l_{\mathrm{c}}$, and $l_{\mathrm{s}}$ will determine the morphology of the surface-induced structures. It also follows that the response to applied force measured in terms of force-extension profiles will depend on $l_{\mathrm{p}}, l_{\mathrm{c}}$, and $l_{\mathrm{s}}$. In this paper, we explore a range of values of $k_{\mathrm{B}} T$ and $l_{\mathrm{s}}$ to predict the force-extension curves for semiflexible chains in poor solvents.

\section{Theory}

Consider a semiflexible chain interacting with a flat surface with the SC-surface potential being $U_{\text {ads }}$. Force $\mathbf{f}=f \cdot \mathbf{n}_{x}$ is applied to one end of the chain (Figure 1). The equilibrium chain configuration is described using the conditional probability $G\left(\mathbf{x}_{N}\right.$, $\left.\mathbf{x}_{1} ; \mathbf{f}\right)$ of finding the tagged $N$ th monomer at $\mathbf{x}_{N}$ given that the monomer, $\mathbf{x}_{1}$, is anchored at the surface, where $\mathbf{x}=(\mathbf{r}, \mathbf{n})$ includes position vector $\mathbf{r}=(x, y, z)$ and orientation vector $\mathbf{n}$, respectively. Because of axial symmetry, the free end orientation is specified by the angle $\theta$ between its tangent vector and the $x$ axis and distance from the surface, $x$ (Figure 1), and the conditional probability $G\left(x_{N}, \theta_{N} ; x_{1}, \theta_{1} ; f\right)$ can be used instead of $G\left(\mathbf{x}_{N}, \mathbf{x}_{1} ; \mathbf{f}\right)$. In the limit $L \rightarrow \infty, G\left(x_{N}, \theta_{N} ; x_{1}, \theta_{1} ; f\right)$ is dominated by the ground state, $\psi_{0}$, so that

$$
G\left(x_{N}, \theta_{N} ; x_{1}, \theta_{1} ; f\right) \approx \psi_{0}\left(x_{1}, \theta_{1} ; f\right) \psi_{0}\left(x_{N}, \theta_{N} ; f\right) \exp \left[-\beta N \epsilon_{0}\right]
$$

$\epsilon_{0}=E_{0} / N$ is the equilibrium free energy per monomer and $\beta=$ $1 / k_{\mathrm{B}} T$. If the SC is modeled as a wormlike chain (WLC), then $\psi_{0}$ satisfies $^{22,27,28}$

$$
\begin{array}{r}
-2 l_{\mathrm{p}} \gamma \frac{\partial \psi_{0}}{\partial x}+\left(1-\gamma^{2}\right) \frac{\partial^{2} \psi_{0}}{\partial \gamma^{2}}-2 \gamma \frac{\partial \psi_{0}}{\partial \gamma}+\beta l_{\mathrm{p}} \gamma f \psi_{0}= \\
\beta\left(u_{\mathrm{ads}}(x)-\epsilon_{0}\right) \psi_{0}
\end{array}
$$

where $u_{\text {ads }}=U_{\text {ads }} / N$ is the adsorption potential per monomer and $\gamma=\cos [\theta]$ (see Figure 1). To mimic the Lennard-Jones chain-surface attractive interaction used in the Langevin simulations (see Section III), we employ a piecewise continuous potential, that is, $u_{\text {ads }}=\infty$ for $x<0, u_{\text {ads }}=-\Delta$ for $0 \leq x \leq b$ and $u_{\text {ads }}=0$ for $x>b$. The monomer density of the adsorbed structures in the absence of force

$$
n(x)=\int \mathrm{d} \theta \psi_{0}^{2}(x, \theta)
$$

normalized as $\int \mathrm{d} x n(x)=N$, is calculated by solving eq 2 without the last term $\beta l_{\mathrm{p}} \gamma f \psi_{0}$.

The perturbative solution of eq 2 in the absence of the $\beta l_{\mathrm{p}} \gamma f$ term, due to Kuznetsov and Sung, ${ }^{27}$ to the first order in correlation length parameter $\eta=\left(4 l_{\mathrm{p}}^{2} / \psi_{0}\right)\left|\mathrm{d}^{2} \psi_{0} / \mathrm{d} x^{2}\right|$ is outlined in Appendix A. The solution is

$$
\begin{aligned}
\psi_{0}(x) & =h(b-x)\left(C_{1} \sin \left(\frac{x \sqrt{m_{1}}}{2 l_{\mathrm{p}}}\right)+C_{2} \sin \left(\frac{x \sqrt{m_{2}}}{2 l_{\mathrm{p}}}\right)\right. \\
& \left.+C_{3} \cos \left(\frac{x \sqrt{m_{1}}}{2 l_{\mathrm{p}}}\right)-C_{3} \cos \left(\frac{x \sqrt{m_{2}}}{2 l_{\mathrm{p}}}\right)\right), \\
& \psi_{0}(x)=C_{0} h(x-b) \exp \left(-\frac{x \sqrt{k}}{2 l_{\mathrm{p}}}\right)
\end{aligned}
$$

where $h(x)$ is the Heaviside function, and $C_{0}, C_{1}, C_{2}$, and $C_{3}$ are constant coefficients; $m_{1}, m_{2}$, and $k$ are given by

$$
\begin{gathered}
m_{1,2}=\frac{15}{8}\left(2+\phi_{\text {in }}\right)\left(6+\phi_{\text {in }}\right)\left(1 \mp \sqrt{1+\frac{16}{5} \frac{\phi_{\text {in }}}{6+\phi_{\text {in }}}}\right) \\
k=\frac{15}{8}\left(2+\phi_{\text {out }}\right)\left(6+\phi_{\text {out }}\right)\left(-1+\sqrt{\frac{16}{5} \frac{\phi_{\text {out }}}{6+\phi_{\text {out }}}}\right)
\end{gathered}
$$

and $\phi_{\text {in }}=\beta\left(u_{\text {ads }}(x)-\epsilon_{0}\right)<0, \phi_{\text {out }}=-\epsilon_{0}>0$. By using two continuity requirements (A9) and the normalization, we can obtain, respectively, $\epsilon_{0}$ and $C_{0}$ and one of $C_{1}, C_{2}$, or $C_{3}$. However, the two free constants are to be chosen such that a minimum of $\epsilon_{0}$ is obtained. The minimal free energy corresponding to the ground state for $x \leq b$ is attained for (i) $C_{1} \neq$ $0, C_{2}=C_{3}=0$ (i.e., the state with $m=m_{1}$ ) and (ii) $C_{2} \neq 0, C_{1}$ $=C_{3}=0\left(m=m_{2}\right)$.

The perturbative solution of eq 2 ignores variation of $\psi_{0}$ on $\theta$. Indeed, when $x \gg l_{\mathrm{p}}, \psi$ becomes nearly isotropic, $\psi(x, \theta)$ $=\psi(x)$. However, when $x \approx b \ll l_{\mathrm{p}}, \psi_{0}$ should depend strongly on the angle $\Theta=\pi / 2+\theta$ between the free end of the chain and the surface (Figure 1). In this range $-\gamma \partial / \partial x \rightarrow \Theta \partial / \partial x$, $\left(1-\gamma^{2}\right) \partial^{2} / \partial \gamma^{2} \rightarrow \partial^{2} / \partial \Theta^{2}, \gamma \partial / \partial \gamma \rightarrow 0, \gamma f \rightarrow-\Theta f$ and eq 2 simplifies, that is

$$
\Theta \frac{\partial \psi_{0}}{\partial x}+\frac{1}{2 l_{\mathrm{p}}} \frac{\partial^{2} \psi_{0}}{\partial \Theta^{2}}-\frac{1}{2} \Theta f \psi_{0}=\frac{\beta}{2 l_{\mathrm{p}}}\left(u_{\mathrm{ads}}(x)-\epsilon_{0}\right) \psi_{0}
$$

The methodology for solving eq 6 has been presented by Semenov in ref 28 and is outlined in Appendix B. The general solution for $x>b\left(u_{\mathrm{ads}}=0\right)$ is

$$
\psi_{0}(x, \Theta)=\sum_{n=0,1} C_{n} x^{1 / 6-n} \Psi\left(n-\frac{1}{6}, \frac{2}{3}, \frac{2 l_{\mathrm{p}} \Theta^{3}}{9 x}\right)
$$

where $C_{0}$ and $C_{1}$ are constants and the confluent hypergeometric function $\Psi(\chi, \omega, z)$ is $\Psi(\chi, \omega, z) \equiv 1 / \Gamma(\chi) \int_{0}^{\infty} \mathrm{d} \tau \tau^{\chi-1}(1+$ 
$\tau)^{\omega-\chi-1} \mathrm{e}^{-\tau z}$ where $\Gamma(\chi) \equiv \int_{0}^{\infty} \mathrm{d} \tau \tau^{\chi^{-1}} \exp [-\tau]$ is a Gamma function. ${ }^{29}$ To describe the chain in the range $x \leq b$, we assume that $\psi_{0}$ is of the form of eq 7 and $C_{0}$ and $C_{1}$ depend on $x$. Substituting eq 7 into eq 6 , we obtain

$$
\begin{aligned}
& \frac{\mathrm{d} C_{0}}{\mathrm{~d} x}=\phi(x)\left(\frac{2 l_{\mathrm{p}}}{x}\right)^{1 / 3}\left(F_{00} C_{0}+F_{01} x^{-1} C_{1}\right) \\
& \frac{\mathrm{d} C_{1}}{\mathrm{~d} x}=\phi(x)\left(\frac{2 l_{\mathrm{p}}}{x}\right)^{1 / 3}\left(F_{10} x C_{0}+F_{11} C_{1}\right)
\end{aligned}
$$

where $F_{n m} \equiv\left(g_{m} / \kappa, f_{n}\right) /\left(f_{n}, f_{n}\right), n, m=0,1$, and $\left(g_{n}, g_{m}\right) \equiv$ $\int_{-\infty}^{\infty} \mathrm{d} \kappa \kappa \mathrm{e}^{-\kappa^{3} / 9} g_{n}(\kappa) g_{m}(\kappa)$ (see eq B2). We solve eq 8 subject to the condition $\phi(x)=\phi_{\text {in }}$ for $x \leq b$ and $\phi(x)=\phi_{\text {out }}$ for $x>b$. From the solutions of eq 8 in Appendix B we obtain:

$$
\begin{aligned}
C_{1}^{\text {in }}(x) & =c_{2} \mathrm{e}^{3 / 2 \sqrt{-D} x^{2 / 3}}\left(-3 \sqrt{-D} x^{2 / 3}\right)^{3 / 2} \\
& \times \Phi\left(\rho+\frac{3}{2}, \frac{5}{2},-3 \sqrt{-D} x^{2 / 3}\right) h(b-x) \\
C_{1}^{\text {out }}(x) & =c_{1} \mathrm{e}^{-3 / 2 \sqrt{-D} x^{2 / 3}} \Phi\left(\rho,-\frac{1}{2}, 3 \sqrt{-D^{2 / 3}}\right) h(x-b)
\end{aligned}
$$

where $\sqrt{-D}=\phi_{\text {out }}\left(2 l_{\mathrm{p}}\right)^{1 / 3} \sqrt{F_{01} F_{10}-F_{11} F_{00}}$. In eq 9, the Kummer function $\Phi(k, l, x)$ is defined by $\Phi(k, l, x) \equiv 1+\sum_{m=1}^{\infty}$ $(k)_{m} /(l)_{m} x^{m} / m$ ! with $(k)_{0}=1,(k)_{1}=k$ and $(k)_{m}=k(k+1) \ldots(k$ $+m-1) .{ }^{29}$ We get $C_{0}(x)$ by substituting eq 9 for $C_{1}^{\text {in }}$ and $C_{1}^{\text {out }}$ into the second eq 8 and $\psi_{0}(x, \Theta ; f)$ can now be obtained by using eq 7 .

In the presence of pulling force, $\psi_{0}$ is nearly isotropic, that is, $\psi_{0}(x, \theta ; f) \approx \psi_{0}(x ; f)$. This allows us to analyze forceextension profiles by employing the perturbative treatment outlined above. Solution to eq 2 is given by

$$
\psi_{0}(x ; f)=\psi_{0}(x) \mathrm{e}^{1 / 2 \beta f x}
$$

where $\psi_{0}(x)$ is given by eq 4 . The average extension as a function of applied force can be computed using

$$
\langle x(f)\rangle \equiv \frac{1}{\beta} \frac{1}{Z(f)} \frac{\mathrm{d}}{\mathrm{d} f} Z(f)
$$

where partition function $Z(f)$ is $Z(f)=\int \mathrm{d} \theta_{N} \int \mathrm{d} \theta_{1} \int \mathrm{d} x_{N} \int \mathrm{d} x_{1}$ $G\left(x_{N}, \theta_{N} ; x_{1}, \theta_{1} ; f\right)$.

The perturbation theory is strictly valid only when the condensation length $l_{\mathrm{c}} \gg l_{\mathrm{p}}$. In practice, we find that the firstorder perturbation theory gives results that are in very good agreement with simulations even when $l_{\mathrm{c}} \approx l_{\mathrm{p}}$. Kuznetsov and Sung also discovered that the perturbation theory is remarkably successful outside the regime of applicability. ${ }^{27}$

\section{Langevin Dynamics Simulations}

We model a semiflexible chain (SC) by $N=100$ connected beads of bond length $a$ and the contour length $L=100 a$. In the absence of $U_{\text {ads }}$ and $f=0$, we assume that the dynamics is governed by the overdamped Langevin equation

$$
\xi \frac{\mathrm{d}}{\mathrm{d} t} \mathbf{x}_{j}=-\frac{\partial U}{\partial \mathbf{x}_{j}}+\mathbf{g}_{j}(t)
$$

where $\xi$ is the friction coefficient, $U=U_{\text {chain }}=U_{\text {bond }}+U_{\text {bend }}$ $+U_{L J}$ is the chain internal energy due to bond potential $U_{\text {bond }}$, bend potential $U_{\text {bend, }}$ and interbead interaction potential $U_{\mathrm{LJ}}$ (hydrodynamic interactions are ignored). The random force $\mathbf{g}_{j}(t)$ obeys Gaussian statistics

$$
\left\langle\mathbf{g}_{j}(t)\right\rangle=0, \quad\left\langle\mathbf{g}_{i}(t) \mathbf{g}_{j}\left(t^{\prime}\right)\right\rangle=6 k_{\mathrm{B}} T \xi \delta_{i j} \delta\left(t-t^{\prime}\right)
$$

We solve eq 12 for each $\mathbf{x}_{j}$ with unit tangent vector $\mathbf{u}_{j}=$ $\left(\mathbf{x}_{j+1}-\mathbf{x}_{j}\right) / a$, where $j=1,2, \ldots, N$. The stretching potential, $U_{\text {bond }}$, is

$$
U_{\text {bond }}=\frac{A}{2 \sigma^{2}} \sum_{j=1}^{N-1}\left(\left|\mathbf{x}_{j}-\mathbf{x}_{j+1}\right|^{2}-\sigma\right)^{2},
$$

where $A$ and $\sigma$ are constants, and

$$
U_{\text {bend }}=\frac{S^{N-1}}{2} \sum_{j=1}^{N}\left(1+\cos \left[\varphi_{j, j+1}\right]\right)^{2}
$$

where the constant $S$ is a measure of chain stiffnes, and $\cos \left[\varphi_{j, j+1}\right]=\left(\mathbf{x}_{j+1}-\mathbf{x}_{j}\right)\left(\mathbf{x}_{j-1}-\mathbf{x}_{j}\right) / \sigma^{2}$ is the bend angle. The interaction between beads is given by the 12-6 Lennard-Jones potential

$$
U_{\mathrm{LJ}}=B \sum_{i<j}\left[\left(\frac{\sigma}{\Delta \mathbf{x}_{i j}}\right)^{12}-2\left(\frac{\sigma}{\Delta \mathbf{x}_{i j}}\right)^{6}\right]
$$

where $\Delta \mathbf{x}_{i j}$ is the distance between beads $i$ and $j$, and $B$ is the magnitude of interaction. $U_{\mathrm{LJ}}$ is an effective interaction that accounts for excluded volume interactions and counterion induced attraction, which in DNA is due to screening of the charges. The persistence length of the chain, $l_{\mathrm{p}}$, can be roughly estimated by using $l_{\mathrm{p}}=a /\left(1-\cos \left[\left\langle\varphi_{j, j+1}\right\rangle\right]\right)$ where $\left\langle\varphi_{j, j+1}\right\rangle=$ $(N-1)^{-1} \sum_{j=1}^{N-1} \varphi_{j, j+1}$ is the average angle between adjacent beeds.

Similar models have been used in previous studies to probe the chain collapse in poor solvents. ${ }^{20,26}$ In the presence of the adsorbing surface, the motion of $j$ th bead is governed by eq 12 with $U=U_{\text {chain }}+U_{\text {ads }}$, where $U_{\text {ads }}$ is the surface-SC potential

$$
U_{\mathrm{ads}}=\Delta \sum_{i}\left[\left(\frac{b}{\Delta \mathbf{x}_{i}}\right)^{12}-2\left(\frac{b}{\Delta \mathbf{x}_{i}}\right)^{6}\right]
$$

In eq $17, \Delta \mathbf{x}_{i}$ is the bead-surface distance and $\Delta$ and $b$ are, respectively, the depth and range of the attractive forces. We set $B=1.0, \sigma=a=1$ and $b=3 a$, and use $A=400 B, S=$ $30 B, 60 B$, and $120 B$ and $\Delta=1.5 B, 2.0 B$, and $2.5 B$. This makes $U_{\mathrm{LJ}}, U_{\text {bond }}, U_{\text {bend }}$, and $U_{\text {ads }}$ to scale in units of $\epsilon_{h}=k_{\mathrm{B}} T$ and $\epsilon_{l}$ $=\sigma$ is the unit length. The choice $A=400 B$ allows for $5 \%$ of thermal fluctuations in the bond distance and permits us to run simulations with longer time steps without affecting bond relaxation time. The unit of time is $\tau=\xi \sigma^{2} / \epsilon_{h}$, where $\xi=44.0$ is the friction coefficient of the chain in water at $T=300 \mathrm{~K}$. The system of eq 12 is integrated with a step size $\delta t=2 \times$ $10^{-2} \tau$ and the total time is $t=N_{\text {tot }} \delta t$ where $N_{\text {tot }}$ is the number of integration steps. We express time either in units of $\tau$ or in terms of $N_{\text {tot }}$.

\section{Results}

A. Surface-Induced Structural Transitions. It is known that in the absence of the surface SC undergoes a collapse transition when the solvent is poor, that is, when the attractive monomermonomer interactions dominate (eq 16) so that $l_{\mathrm{c}}>l_{\mathrm{p}}$. The collapse is a result of a competition between intramolecular attraction and bending energy due to chain stiffness. Unlike in 


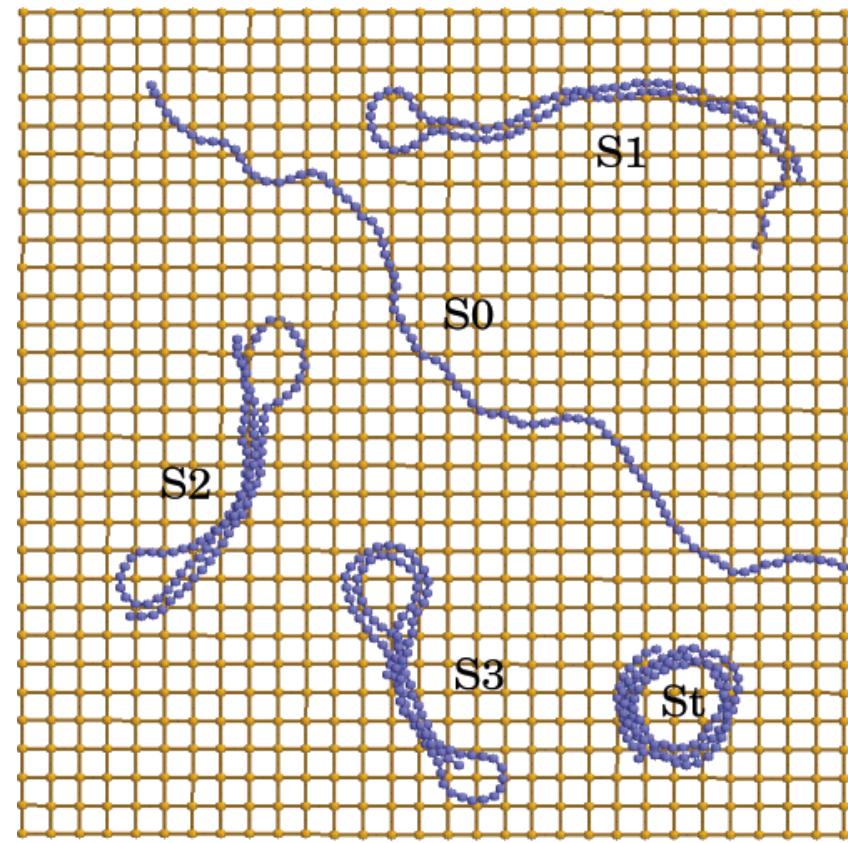

Figure 2. Top view of the typical structures (blue) adsorbed on the surface (yellow) for $S=120 B, \Delta=1.5 B$, and $k_{\mathrm{B}} T=1.0$. Extended $\left(S_{0}\right)$, one-racquet $\left(S_{1}\right)$, two-racquet $\left(S_{2}\right)$, three-racquet $\left(S_{3}\right)$, and toroidal structures $\left(S_{\mathrm{t}}\right)$ are obtained in a single trajectory that is terminated at $t$ $=4 \times 10^{-5} \tau$. The equilibrium structure under these conditions is the toroid.

flexible polymers, the low energy collapsed conformation is a torus that maximizes intramolecular contacts and minimizes the bending penalty. Before simulating the force-extension curves of adsorbed SC, it is necessary to characterize the structures that are obtained when interacting with the surface.

To simulate the low free-energy structures that result in the presence of the surface, we first thermalized an extended chain at high temperature $k_{\mathrm{B}} T=3.0$ for $N_{\text {tot }}=1 \times 10^{6}$ steps. By gradually decreasing the temperature, bulk structures were thermalized for $(1-10) \times 10^{7}$ steps and used in adsorption experiments. Interactions with the attractive surface was switched on at distance $\Delta x=2 b$ away from the bead with shortest $x$, and the SC was adsorbed onto the surface one bead at a time. The structures were allowed to relax for $\sim 1-20 \times 10^{6}$ steps depending on $k_{\mathrm{B}} T, S$, and $\Delta$. The progress of adsorption was monitored by analyzing time traces of $U_{\mathrm{LJ}}, U_{\mathrm{ads}}, U$, and the radius of gyration, $R_{\mathrm{g}}$, of the $\mathrm{SC}$. We generated 500 adsorbed structures at $k_{\mathrm{B}} T=1.0,1.25$, and 1.5 for $S=30 B, 60 B$, and $120 \mathrm{~B}$ and $\Delta=1.5 \mathrm{~B}, 2.0 \mathrm{~B}$, and $2.5 \mathrm{~B}$.

Typical structures are presented in Figure 2. The geometry of the SC adsorbed onto the surface ranges from partially or fully extended configuration with $l_{\mathrm{p}} / a \approx 18$ to partially structured one-, two- and three-racquet states with $l_{\mathrm{p}} / a \approx 16.0,15.5$, and 15.0 , respectively, to fully ordered toroidal states with $l_{\mathrm{p}} / a \approx$ 13.5. Similar structures have been observed in recent studies of collapse of semiflexible chains in the bulk. ${ }^{20,26}$ For the interaction parameters used in our simulations, $l_{\mathrm{s}} / l_{\mathrm{c}} \approx o(1)$. Thus, the attractive $\mathrm{SC}$-surface interaction facilitates adsorption of the SC without significantly altering its morphology compared to the bulk case. For $l_{\mathrm{s}} \gg l_{\mathrm{c}}$, the lowest free-energy structures are extended.

To compare the kinetics of structure formation on the surface and in the bulk, we also simulated collapsed structures in the absence of the adsorbing surface. By analyzing the temporal profiles of $R_{\mathrm{g}}, U_{\mathrm{LJ}}$, and $l_{\mathrm{p}}$, we found that on average, chains attain structured configurations on a faster time scale when adsorbed on the surface. The search for the ground (toroidal) state is more efficient when the chain is constrained to evolve on the 2D surface where the SC quickly minimizes its free energy in reduced $d=2$ space by sliding surface motion (lateral diffusion).

B. Kinetics of Surface-Induced Ordering. Typically, surfaceinduced ordered structures form by a two step process $B_{0} \rightarrow S_{0}$ $\rightarrow S_{\mathrm{t}}$. Starting from the bulk state $B_{0}$, extended surface transient $S_{0}$ emerges during the fast first step with the $B_{0} \rightarrow S_{0}$ transition occurring within $N_{\text {tot }}=1-3 \times 10^{6}$. In the slower second step, $S_{0} \rightarrow S_{\mathrm{t}}$, extended transient structures explore the free-energy landscape in search of the toroidal state, $S_{\mathrm{t}}$, which occurs in about $3-20 \times 10^{6}$ steps depending on $S, \Delta$, and temperature. Transition from $S_{0}$ to $S_{\mathrm{t}}$ is realized via rapid formation of either a surface loop or an intermediate toroid-like motif with larger $R_{\mathrm{g}}$ (smaller winding number) or through a sequence of longer lived racquet states $S_{0} \rightarrow S_{1} \rightarrow \ldots \rightarrow S_{\mathrm{t}}$, where $S_{n}, n=0,1,2$, ... denotes conformations with number of racquets equal to zero (extended chain) one, two, and so forth.

The number of "metastable" racquets depends on the chain flexibility. We observed configurations with $n=6$ for $S=30 B$ and $k_{\mathrm{B}} T=1.25$. Simulated profiles of $R_{\mathrm{g}}, U_{\mathrm{LJ}}, U_{\text {ads }}$, and $U$ indicate that evolution from extended to toroidal states follows several pathways. Four out of five simulation runs followed the scheme outlined above. Similar diverse pathways have been observed by Noguchi and Yoshikawa ${ }^{20}$ who recorded the lifetime of intermediates species for about $N_{\text {tot }}=2.0 \times 10^{5}$. Our results indicate that attractive surface forces increase the lifetimes of metastable intermediates for stiff chains at low temperature. In a few simulation runs, toroidal structures were not observed during as many as $20 \times 10^{6}$ steps. Hence, attractive surface forces facilitate the formation of toroidal state primarily when formation of toroid-like intermediate motif is involved.

The dynamics of $R_{\mathrm{g}}, U_{\mathrm{LJ}}, U_{\mathrm{ads}}$, and $U$ for the structures in Figure 2 show that increasingly more ordered states are also energetically favorable (Figure 3 ). $R_{\mathrm{g}}, U$, and $U_{\mathrm{LJ}}$ decrease and $U_{\text {abs }}$ increases in the sequence $S_{0} \rightarrow S_{1} \rightarrow S_{2} \rightarrow S_{3} \rightarrow S_{\mathrm{t}} . R_{\mathrm{g}}$ fluctuates around larger values for extended states. Variations in $U_{\mathrm{LJ}}, U_{\mathrm{ads}}$, and $U$ increasing in the sequence of $S_{1} \rightarrow S_{2} \rightarrow S_{3}$ $\rightarrow S_{t}$ transitions are due to the formation of $\mathrm{SC}$-surface contacts. For the structures in Figure 2, the formation of $S_{1}$ at $N_{\text {tot }} \approx 2.0$ $\times 10^{6}$ is mediated by a surface-loop motif followed by slow sliding motion; $S_{2}$ forms early at $N_{\text {tot }} \approx 5.0 \times 10^{5}$ and remains unchanged (time dependence of $R_{\mathrm{g}}$ or $U_{\mathrm{LJ}}$ ). The dynamics of $U_{\mathrm{LJ}}, U_{\mathrm{ads}}$, and $U$ show formation of $S_{3}$ via $S_{1}$ at $N_{\text {tot }} \approx 1.0 \times$ $10^{5}$ followed by transition $S_{1} \rightarrow S_{3}$ at $N_{\text {tot }} \approx 7.0 \times 10^{6}$. Similarly, traces of the same quantities for $S_{\mathrm{t}}$ point at three-step transition, $S_{0} \rightarrow S_{1} \rightarrow S_{3} \rightarrow S_{\mathrm{t}}$ occurring, respectively, at $N_{\text {tot }} \approx 5.0 \times 10^{5}$, $1.5 \times 10^{6}$, and $4.0 \times 10^{6}$, followed by chain compaction due to sliding motion.

In agreement with theoretical arguments, ${ }^{28}$ monomer profiles of stiff chains $\left(S=120 B, l_{\mathrm{p}} / b \gg 1\right)$ are described by the succession of short near-surface loops of length $\ll l_{\mathrm{p}} / a$ between chain-surface contact and by the combination of short and long loops of the length $\gg l_{\mathrm{p}} / a$ for $S=30 B$ and $l_{\mathrm{p}} / b \approx 1$. Decrease in $l_{\mathrm{p}} / b$ and temperature favors the formation of chain-surface contacts by enabling more beads to be inside the range of surface forces. This results in the formation of higher ordered states $S_{4}, S_{5}, S_{6}$, and $S_{\mathrm{t}}$. In contrast, at higher temperatures and increased $\Delta$ and $S$, surface structures with increased conformational free energy become unstable and unfold into extended configurations (data are not shown). We quantified the geometry of surface structures (Figure 2) by binning bead-surface distances, $x_{j}$, into the density histogram, $n(x)$. The monomer 

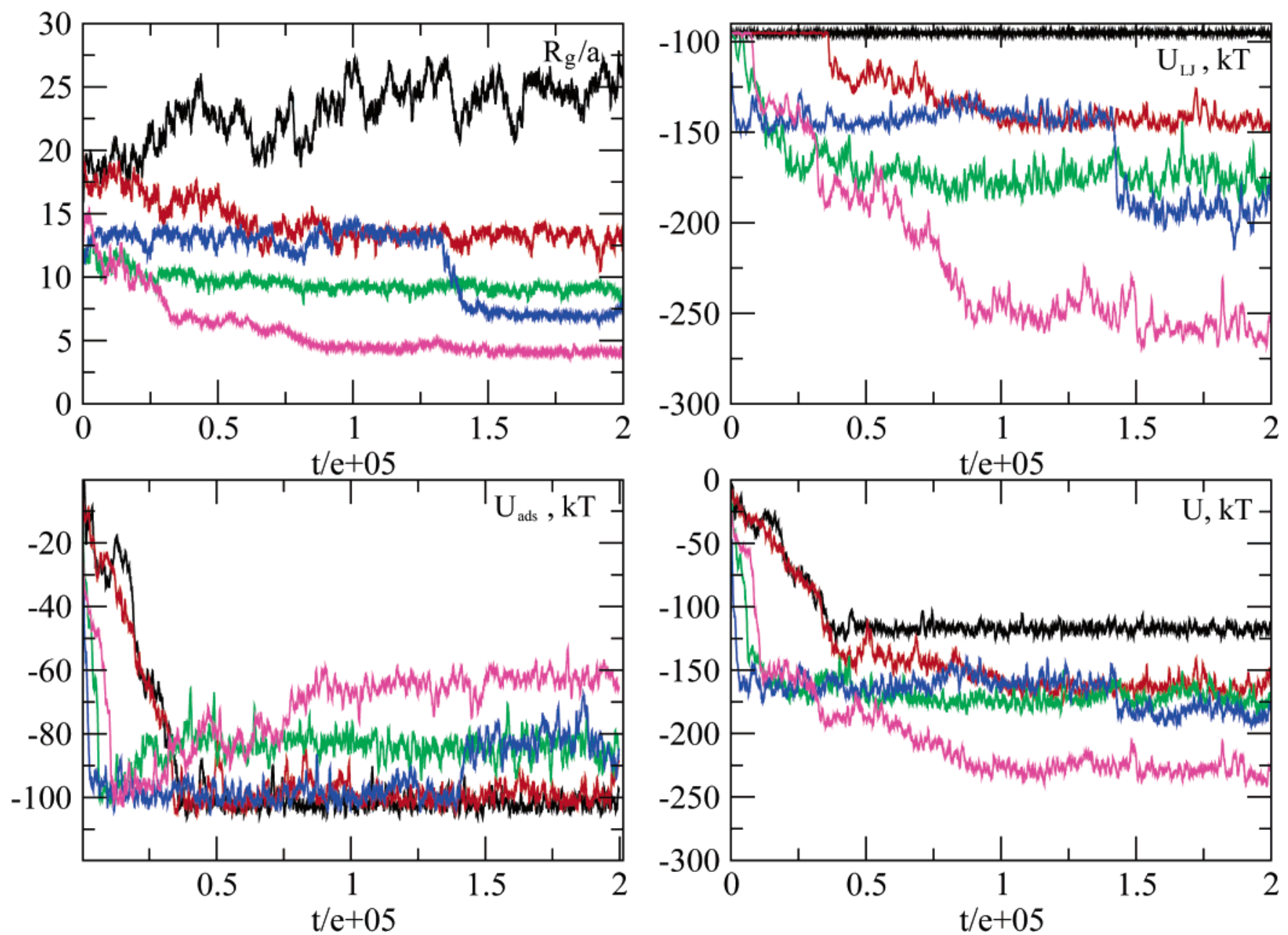

Figure 3. Dependence of radius of gyration, $R_{\mathrm{g}} / a$ (top left), intramolecular attractive interaction, $U_{\mathrm{LJ}}$ (eq 16), surface potential, $U_{\text {ads }}$ (eq 17), and internal energy, $U\left(=U_{\text {bend }}+U_{\text {bond }}+U_{\mathrm{LJ}}\right)$ displayed as functions of time, measured in units of $\tau$. The five curves in each panel correspond to extended (black), one-racquet (red), two-racquet (green), three-racquet (blue), and toroidal (magenta) structures of Figure 2.

density profiles for $\Delta=1.5 B$ and $k_{\mathrm{B}} T=1.0$ are compared in Figure 4 for $S=70 B$ (left) and $S=50 B$ (right panels). Transition from less-structured to more-structured states is accompanied by an increased ratio of the number of beadbead to bead-surface contacts. The density distribution, $n(x)$, is single-peaked at $x=b / 2$ and decays to zero as $x \rightarrow b$ for extended states and increases its density at $x \approx b$ in the sequence $S_{1} \rightarrow S_{2} \rightarrow S_{3} \rightarrow S_{\mathrm{t}}$.

C. Forced Unfolding of Surface-Adsorbed Structures. To unfold the surface-ordered structures, these structures were initially allowed to thermalize at $k_{\mathrm{B}} T=1.0$ for $N_{\text {tot }}=2 \times 10^{6}$. We then ancored the $C$ terminus of the chain at the surface and pulled its $N$ terminus with constant force $f$ via the harmonic spring with the spring constant $k_{\mathrm{sp}}=0.36 \mathrm{pNnm}^{-1}$ in the direction perpendicular to the surface. Simulation runs were terminated after evolution of chain extension $x\left(N_{\text {tot }}\right)$ had reached equilibrium. $x\left(N_{\mathrm{tot}}\right)$ of the structures of Figure 2 are presented in Figure 5 for $f=9.75,18.3,24.4$, and $30.5 p N$. Chain extension reaches a saturation plateau in the first $8 \times 10^{7}$ steps as the chain restoring force approaches $f$. Not unexpectedly, the unfolding threshold force increases with the extent of ordering in the sequence $S_{0} \rightarrow S_{1} \rightarrow S_{2} \rightarrow S_{3} \rightarrow S_{\mathrm{t}}$. At $f=9.75$ $p N$, only $S_{0}$ unfolds in $1.0 \times 10^{7}$ steps. When the force is increased to $f=18.3 p N, S_{0}, S_{1}, S_{2}$, and $S_{3}$ unbinds from the surface in $3.5 \times 10^{7}, 3.6 \times 10^{7}, 4.0 \times 10^{7}$, and $6.0 \times 10^{7}$ steps, respectively. At $f=24.4 \mathrm{pN}$, all structures reach the stretched state in $2-4 \times 10^{7}$ steps. From the dynamical trajectories of $x$ obtained for $\Delta=1.5 B$ and $k_{\mathrm{B}} T=1.0$, we constructed the average extension, $\langle x\rangle$, as a function of $f$. In Figure 6 we compare $\langle x\rangle$ versus $f$ traces for extended, one-racquet, three-racquet, and toroidal structures of Figure $2(S=120 B$, top panel) and more flexible four-, five-, and seven-racquet and toroidal conformations obtained for $S=30 \mathrm{~B}$. Unbinding of surface-anchored structures undergoes a highly cooperative all-or-none transition as the unfolding force threshold $f=f_{\mathrm{c}}$ is increased from 7.3 to $15.8 p N(S=120 B)$ to $15.9-17.7 p N(S=30 B)$ for more compact racquet and toroidal states.

D. Comparison between Theory and Simulations. We analyzed the simulation results for the monomer density and the averaged extension as a function of the pulling force by using perturbative treatment (see eqs $3-5$ ) in the entire range of $x<L$. For the proximal limit $x \ll l_{\mathrm{p}}<L$ we use the exact expressions in eqs 7 and 9 . The density distributions and forceextension profiles for the extended conformation were approximated by chosing the ground state with $m=m_{1}\left(m_{1}<\right.$ $m_{2}$, see eq 5). The choice $m=m_{1}$ corresponds to isotropic-like unstructured surface state with no preferred orientation of the chain beads. Histograms of structured two-, three-racquet, and toroidal conformations were analyzed with the choice $m=m_{2}$ corresponding to nematic-like ordered states. ${ }^{27}$ To account for the difference between the shape of attractive potential $U_{\text {ads }}$ used in the simulations and the theoretical calculation we used, in the actual fit, the rescaled potential depth $\Delta_{T}=r \Delta_{\text {sim }}$ for the same range $b_{T}=b_{\text {sim }}=1$, where $r=\left(b \Delta_{T}\right)^{-1} \int_{0}^{\infty} \mathrm{d} x u_{\text {ads }}(x)$ is the ratio between volume of Lennard-Jones attractive layer and 

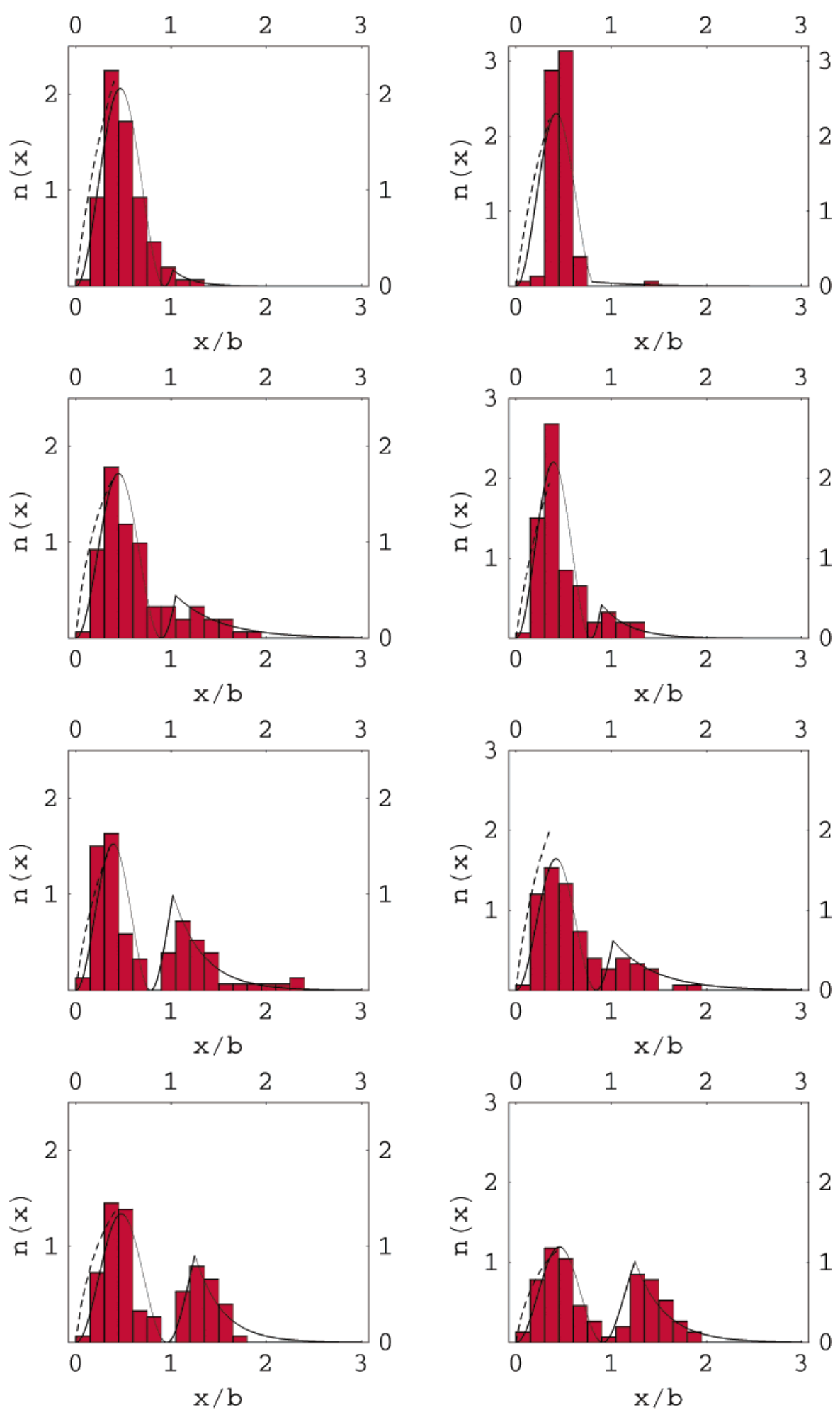

Figure 4. Average monomer density profiles, $n(x)$, as a function of $x / b$ for $\Delta=1.5 B$ and $k_{\mathrm{B}} T=1.0$ for extended (top), two-racquet (top middle), three-racquet (bottom middle), and toroidal states (bottom panel). The left panel is for $S=70 B$, and the results for $S=50 B$ are shown on the right. Solid lines and dotted lines represent the results obtained using perturbative and exact theory, respectively.

$b \Delta_{T}$ used in theory. The density profiles, $n(x)$, for known values of $b, k_{\mathrm{B}} T$, and $\Delta_{T}$ were fitted to the simulated monomer density histograms and force-extension profiles to obtain parameters $\epsilon_{0}$ (eq 1) and $l_{\mathrm{p}}$. The theoretical results for the density, $n(x)$, and the average extension, $\langle x(f)\rangle$, computed from eqs 3 and 11 , respectively, using these parameters are shown in Figures 4 and 6.

Monomer Density Distributions. Although the theoretical results for $n(x)$ slightly underestimate the simulated density for structured states for $0<x / b<0.5$ and overestimates it for $x / b$ $>1.6$, the agreement between perturbation theory and simulation data is surprisingly good in the range of $x / b \approx 1$ (Figure 4 ). The agreement between theory and simulations improves for more structured racquet and toroidal conformations. In particular, the theoretical profiles capture the positions of density peaks both inside the layer at $x / b \approx 0.5$ and at the boundary. Although there is some residual density at large $x / b$ because of thermal fluctuations of chain ends, especially for less structured extended 

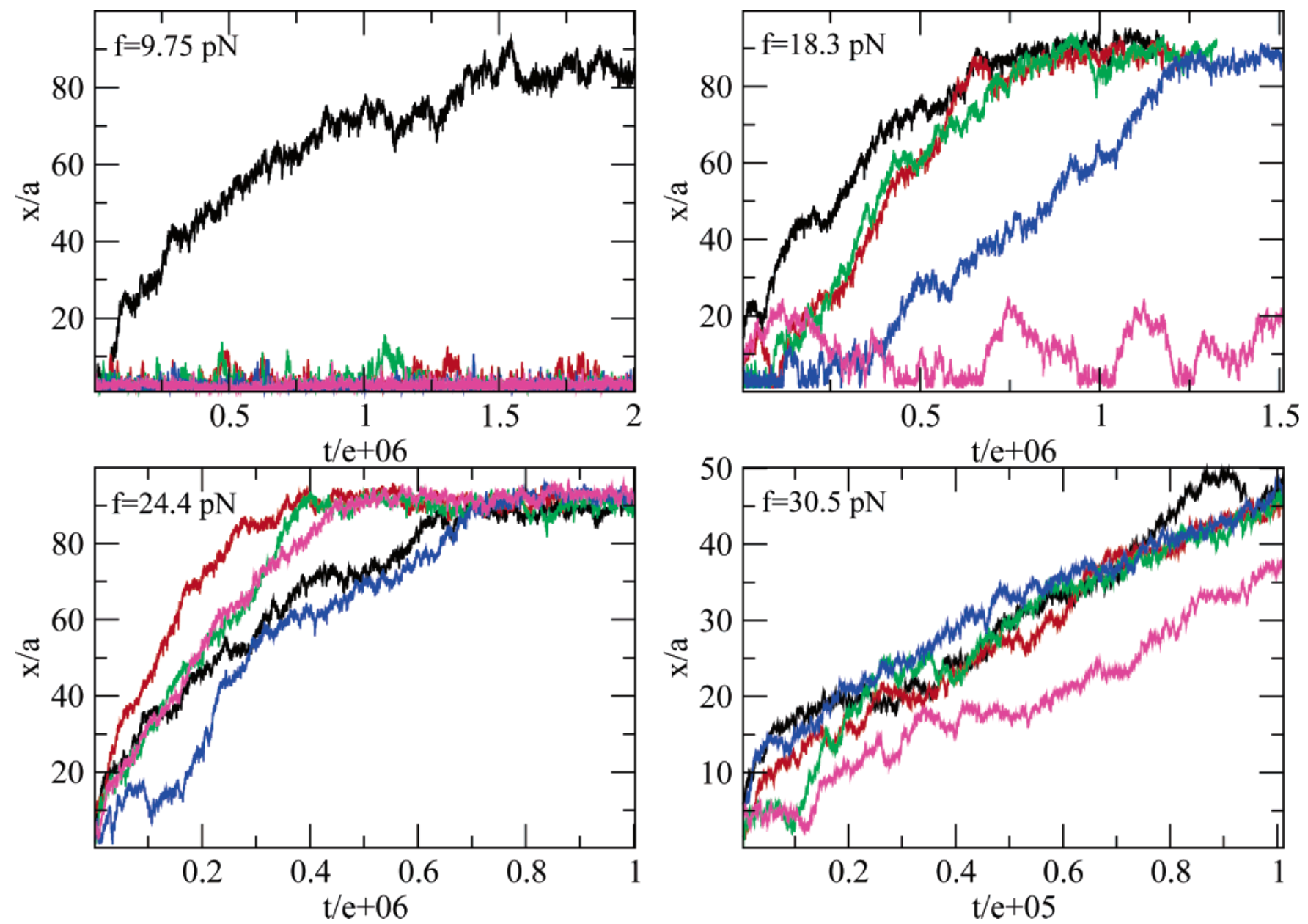

Figure 5. Dynamics of extension $x$ (in units of $a$ ) for a few trajectories at different values of $f$ applied to the chain ends of structures shown in Figure 2. Time $t$ is expressed in units of $\tau$. The colors correspond to the caption in Figure 3. The values of $f$ are displayed in the panels.

and racquet configurations, the ground state dominance approximation is clearly valid. The theoretically estimated conformational free energy per monomer and persistence length for structures $S_{0} \rightarrow S_{2} \rightarrow S_{3} \rightarrow S_{\mathrm{t}}$ of Figure 4 decrease respectively as $\epsilon_{0} / k_{\mathrm{B}} T \approx-0.21 \rightarrow-0.23 \rightarrow-0.24 \rightarrow-0.25$ and $l_{\mathrm{p}} / a \approx 11.7 \rightarrow 11.2 \rightarrow 10.4 \rightarrow 10.2$ (for $k_{\mathrm{B}} T=1.0$, left panels $)$, and $\epsilon_{0} / k_{\mathrm{B}} T \approx-0.23 \rightarrow-0.25 \rightarrow-0.26 \rightarrow-0.27$ and $l_{\mathrm{p}} / a \approx 11.3 \rightarrow 11.0 \rightarrow 10.2 \rightarrow 10.1$ (for $k_{\mathrm{B}} T=1.25$, right panels). Not surprisingly, both $\epsilon_{0}$ and $l_{\mathrm{p}}$ decrease for the same structures as $k_{\mathrm{B}} T$ is increased because of enhanced chain flexibility. In the proximal region, the exact calculation of $n(s)$ for $0 \leq x / b \leq 0.5$ for the same structure sequence shows a better agreement with the simulated results. The fit parameters are $\epsilon_{0} / k_{\mathrm{B}} T \approx-0.2 \rightarrow-0.24 \rightarrow-0.25 \rightarrow-0.28$ and $l_{\mathrm{p}} / a \approx 12.3 \rightarrow$ $11.8 \rightarrow 11.0 \rightarrow 10.8$ for $k_{\mathrm{B}} T=1.0$, and $\epsilon_{0} / k_{\mathrm{B}} T \approx-0.19 \rightarrow$ $-0.22 \rightarrow-0.24 \rightarrow-0.26$ and $l_{\mathrm{p}} / a \approx 12.0 \rightarrow 11.6 \rightarrow 10.8 \rightarrow$ 10.6 for $k_{\mathrm{B}} T=1.25$.

Force-Extension Curves. Apart from small deviations around the unfolding threshold forces for all simulated surface structures, the fit of theoretical curves of the average extension versus pulling force to simulated data points shows excellent agreement between theory and simulations. The theoretical $\langle x(f)\rangle$ curves calculated using the perturbation theory follow closely the simulated force-extension profiles both for $S=120 B$ and $S=$ $30 B$ especially below $(x / L \leq 0.1)$ and above $(x / L \geq 0.9)$. The unbinding threshold forces increase as $7.5 p N<10.5 p N<$ $12.5 p N<16.5 p N$ in the sequence $S_{0} \rightarrow S_{1} \rightarrow S_{3} \rightarrow S_{\mathrm{t}}(S=$ $120 B$, top panel in Figure 6) and as $15 p N<15.5 p N<16.5$ $p N<17.5 p N$ in the sequence $S_{4} \rightarrow S_{5} \rightarrow S_{7} \rightarrow S_{\mathrm{t}}(S=30 B$, bottom panel in Figure 6). This implies that more flexible and/ or more structured surface chains are harder to unfold. However, an "all-or-none" type of simulated unfolding transition shows sharper growth than predicted by the theory. The theoretically estimated conformational free energy per monomer and persistence length for structures $S_{0} \rightarrow S_{1} \rightarrow S_{3} \rightarrow S_{\mathrm{t}}$ decrease, respectively, as $\epsilon_{0} / k_{\mathrm{B}} T \approx-0.12 \rightarrow-0.17 \rightarrow-0.20 \rightarrow-0.22$ and $l_{\mathrm{p}} / a \approx 15 \rightarrow 14.5 \rightarrow 14.25 \rightarrow 12.1$ (top panel). For the structures $S_{4} \rightarrow S_{5} \rightarrow S_{7} \rightarrow S_{\mathrm{t}}, \epsilon_{0}$ decreases as $\epsilon_{0} / k_{\mathrm{B}} T \approx-0.134$ $\rightarrow-0.136 \rightarrow-0.141 \rightarrow-0.148$ and $l_{\mathrm{p}} / a \approx 8.2 \rightarrow 8.1 \rightarrow 8.0$ $\rightarrow 7.9$ (bottom panel). Here too, increased chain flexibility decreases $l_{\mathrm{p}}$ and lowers $\epsilon_{0}$.

\section{Conclusions}

To provide insight into interactions between biomolecules and membranes, we have considered collapse and forced unbinding of semiflexible chains (SC) in the presence of an adsorbing surface. The interaction of SC modeled using WLC, which describes many of the physical properties of DNA, ${ }^{19}$ RNA,${ }^{30}$ and proteins, ${ }^{31}$ with a surface into which the SC can adsorb, is studied using theory and simulations. The morphologies of the SC in the presence of an adsorbing potential is described in terms of three length scales, namely, $l_{\mathrm{p}}, l_{\mathrm{s}}$, and $l_{\mathrm{c}}$. By restricting ourselves to $l_{\mathrm{c}} \approx l_{\mathrm{s}}$, we have studied the effect of interaction with the surface on coil-toroidal transition in DNAlike chains. The simulations show that the rate of toroid formation is impeded compared to the bulk because interaction with the surface stabilizes many metastable racquet-like structures (Figure 1). The simulated equilibrium density profiles show 


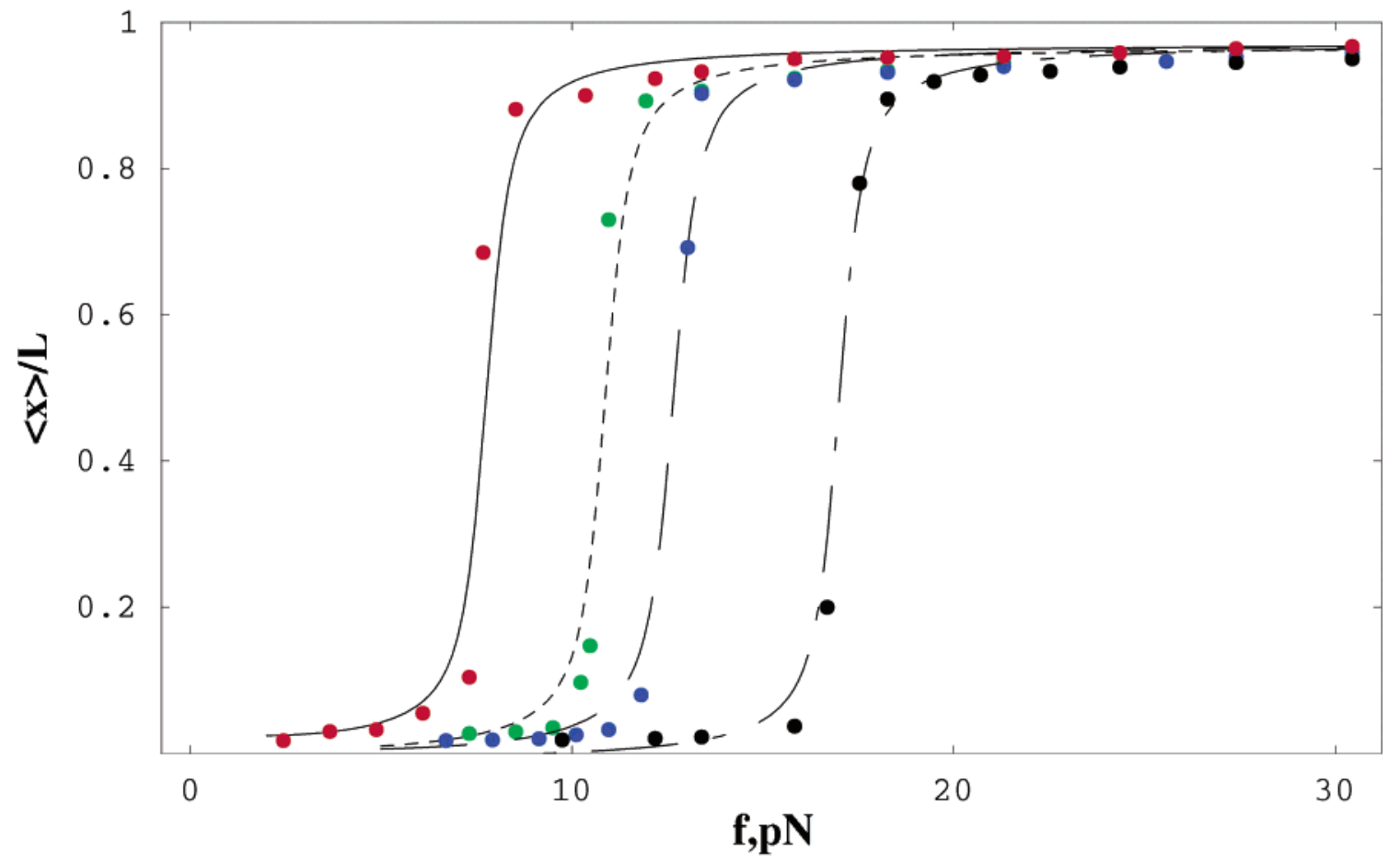

15

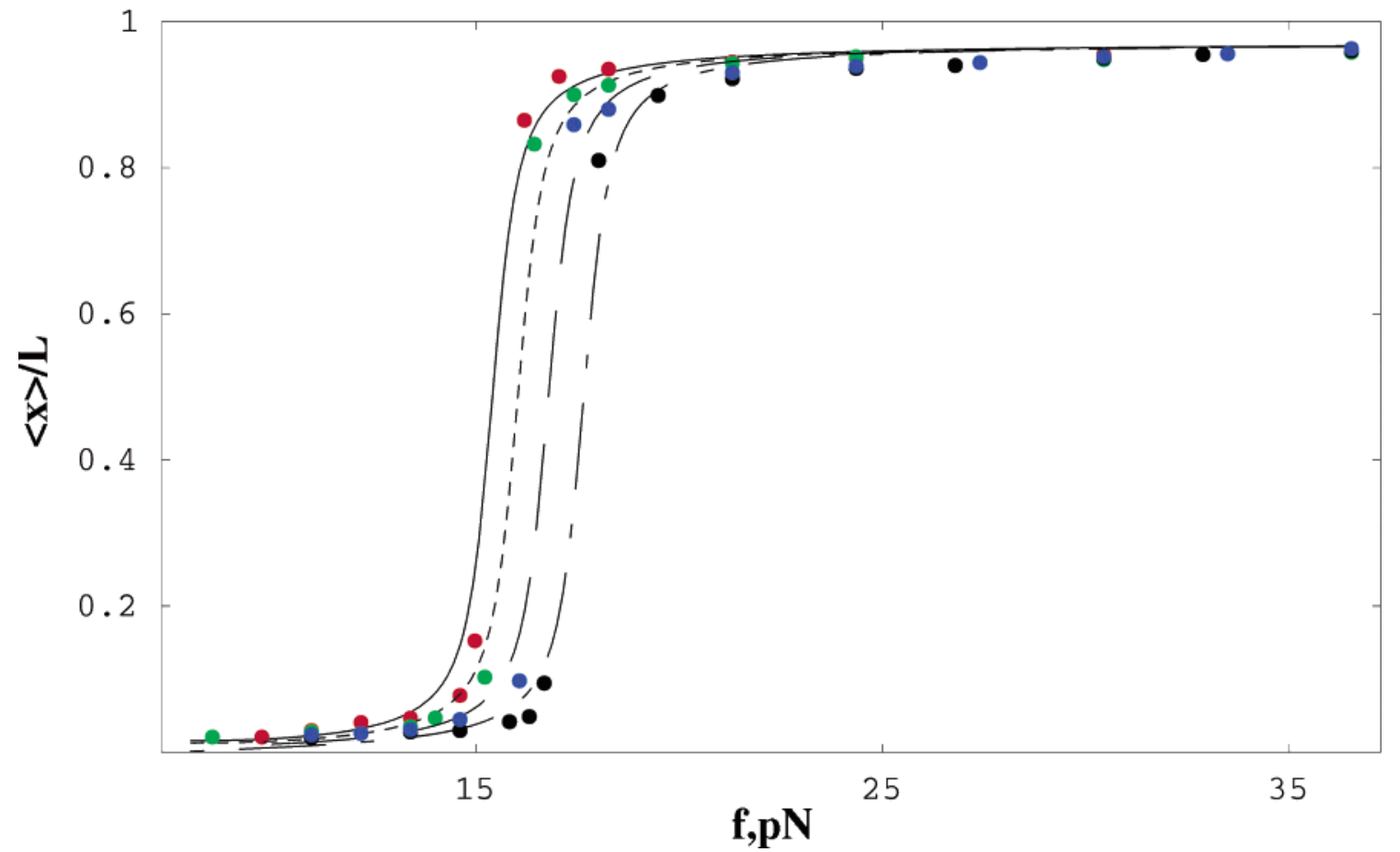

Figure 6. The averaged reduced extension, $\langle x\rangle / L$, as a function of constant force $f$ simulated for $\Delta=1.5 B$ and $k_{\mathrm{B}} T=1.0$ for structures in Figure $2(S=120 B$, top $)$. The bottom panel shows force-extension profiles for four-, five-, seven-racquet, and toroidal configurations obtained for $S=$ $30 B$ (bottom panel). Data points for extended (four-racquet), one-racquet (five-racquet), three-racquet (seven-racquet), and toroidal structures are given by red, green, blue, and black circles, respectively. Theoretical curves for these structures are given, respectively, by solid, dotted, dashed, and dot-dashed lines.

that as the range of surface-SC interaction increases and temperature decreases, which leads to a decrease in $l_{\mathrm{p}} / l_{\mathrm{s}}$, ordered structures form. The peak of $n(x)$ at $x \approx b$ (the range of interaction) grows as $l_{\mathrm{p}}$ decreases. The bimodality in the $n(x)$ distribution function suggests that the surface-induced toroid formation is a first-order transition. The perturbative calculation reproduces qualitatively all of the features in the simulated density profiles.
We also considered the peeling and unbinding of adsorbed structures by applying force. These results, which are of direct relevance to AFM experiments, ${ }^{32}$ show that the forced unbinding transition is surprisingly highly cooperative. For all structures (racquet-like and toroids), unbinding occurs over a narrow force range. The magnitude of the critical force, $f_{\mathrm{c}}$, for a fixed value of $T$ and $l_{\mathrm{s}}$ increases as $l_{\mathrm{p}}$ decreases. From general considerations, we expect that $f_{\mathrm{c}}$ should be described by a scaling 
function $g(y)$ where $y=l_{\mathrm{s}} / l_{\mathrm{p}}$ for a fixed $T$. When $y<y_{\mathrm{c}}$ (a critical value), then adsorption is not free energetically favored. When $y>y_{\mathrm{c}}$, then $f_{\mathrm{c}}$ should increase by an increasing function of $y$. The increase in $f_{\mathrm{c}}$ can be achieved either by increasing $l_{\mathrm{s}}$ for a fixed $l_{\mathrm{p}}$ or by decreasing $l_{\mathrm{p}}$ for fixed $l_{\mathrm{s}}$. Additional work is required to elucidate the nature of the scaling function $g(y)$. Quite surprisingly, we find that the force-extension profiles can be calculated by using a simple perturbation theory even though the nature of the unbinding transition is abrupt. The present work shows that global properties of force-extension characteristics of adsorbed biomolecules can be nearly quantitatively predicted using the proposed theory.

It is now well established that elastic response of DNA, in the absence of interaction with surfaces, depends sensitively on the nature and concentration of counterions. ${ }^{32,33}$ Our work shows that the force-extension curves in the presence of a surface to which DNA is bound depends not only on $l_{\mathrm{s}}$ but also on the morphology of the adsorbed structures. The novel prediction that forced unbinding should occur cooperatively by a first-order phase transition can be probed using single-molecule experiments.

Acknowledgment. This work was supported in part by a grant from the National Science Foundation through NSF CHE05-14056. We would like to thank Changbong Hyeon for many useful discussions.

Appendix A: Perturbative Treatment of Adsorbed Chain Statistics. We expand $\psi_{0}$ (eq 1) in terms of the Legendre polynomials, that is

$$
\psi_{0}(x, \theta)=\sum_{i=0}^{\infty} \psi_{i}(x) P_{i}(\cos [\theta])
$$

By using the following equations

$$
\begin{aligned}
\frac{\mathrm{d}}{\mathrm{d} \gamma} P_{i}(\gamma) & =-i(i+1) P_{i}(\gamma) \\
P_{1}(\gamma) P_{i}(\gamma) & =\frac{(i+1) P_{i+1}(\gamma)+i P_{i-1}(\gamma)}{2 i+1}
\end{aligned}
$$

we transform eq 2 without term $\beta l_{\mathrm{p}} \gamma f \psi_{0}$ into $(i \geq 0)$

$$
\begin{aligned}
\frac{i(i+1)+\beta\left(u_{\mathrm{ads}}-\epsilon_{0}\right)}{2 l_{\mathrm{p}}} \psi_{i}(x) & =-\frac{i}{2 i-1} \frac{\mathrm{d} \psi_{i-1}(x)}{\mathrm{d} x} \\
& -\frac{i+1}{2 i+3} \frac{\mathrm{d} \psi_{i+1}(x)}{\mathrm{d} x}
\end{aligned}
$$

To the first few orders we have

$$
\begin{gathered}
\psi_{0}=\frac{2 l_{\mathrm{p}}}{3 \beta\left(u_{\mathrm{ads}}-\epsilon_{0}\right)} \frac{\mathrm{d} \psi_{1}}{\mathrm{~d} x} \quad(i=0) \\
\psi_{1}=-\frac{2 l_{\mathrm{p}}}{2+\beta\left(u_{\mathrm{ads}}-\epsilon_{0}\right)} \frac{\mathrm{d} \psi_{0}}{\mathrm{~d} x}-\frac{4 l_{\mathrm{p}}}{5\left(2+\beta\left(u_{\mathrm{ads}}-\epsilon_{0}\right)\right)} \frac{\mathrm{d} \psi_{2}}{\mathrm{~d} x} \\
(i=1) \\
\psi_{2}=-\frac{4 l_{\mathrm{p}}}{3\left(6+\beta\left(u_{\mathrm{ads}}-\epsilon_{0}\right)\right)} \frac{\mathrm{d} \psi_{1}}{\mathrm{~d} x}-\frac{6 l_{\mathrm{p}}}{7\left(6+\beta\left(u_{\mathrm{ads}}-\epsilon_{0}\right)\right)} \frac{\mathrm{d} \psi_{3}}{\mathrm{~d} x}
\end{gathered}
$$

$\psi_{1}$ is given by the second eq A4 with the second term determined by $\psi_{2}$ which is of the order of $8 l_{\mathrm{p}}^{3} \mathrm{~d}^{3} \psi_{0} / \mathrm{d} x^{3}$. Neglecting this order for $\eta \ll 1$ we obtain

$$
\psi_{1} \approx-\frac{2 l_{\mathrm{p}}}{2+\beta\left(u_{\mathrm{ads}}-\epsilon_{0}\right)} \frac{\mathrm{d} \psi_{0}}{\mathrm{~d} x}
$$

Including the second term in the third eq A4 and using eq A5 we obtain the first-order perturbation equation for $\psi_{1}$

$$
\begin{array}{r}
\psi_{1} \approx-\frac{2 l_{\mathrm{p}}}{2+\phi} \frac{\mathrm{d} \psi_{0}}{\mathrm{~d} x}-\frac{4 l_{\mathrm{p}}}{5(2+\phi)} \\
\frac{\mathrm{d}}{\mathrm{d} x}\left[\frac{4 l_{\mathrm{p}}}{3(6+\phi)} \frac{\mathrm{d}}{\mathrm{d} x}\left(\frac{2 l_{\mathrm{p}}}{2+\phi} \frac{\mathrm{d} \psi_{0}}{\mathrm{~d} x}\right)\right]
\end{array}
$$

where $\phi=\beta\left(u_{\text {ads }}-\epsilon_{0}\right)$. By substituting eq A6 into the first eq A4 we arrive at the first-order perturbative equation for $\psi_{0}$

$$
\frac{64 l_{\mathrm{p}}^{4}}{45 \phi(2+\phi)^{2}(6+\phi) \mathrm{d} x^{4}}+\frac{4 l_{\mathrm{p}}^{2}}{3 \phi(2+\phi) \mathrm{d} x^{2}}-\psi_{0}^{2} \psi_{0}=0
$$

For the class of potentials considered here, the physical solution of eq A7 that satisfies the boundary conditions

$\psi_{0}(x=0)=0, \psi_{0}(x \rightarrow \infty) \rightarrow 0,\left.\frac{\mathrm{d}^{n}}{\mathrm{~d} x^{n}} \psi_{0}\right|_{x \rightarrow \infty} \rightarrow 0, n=1,2, \ldots$

continuity requirements at $x=b$

$\psi_{0}(x \rightarrow b-0)=\psi_{0}(x \rightarrow b+0),\left.\frac{\mathrm{d}}{\mathrm{d} x} \psi_{0}\right|_{x \rightarrow b-0}=\left.\frac{\mathrm{d}}{\mathrm{d} x} \psi_{0}\right|_{x \rightarrow b+0}$

and normalization condition is given by eq 4 .

Appendix B: Exact Treatment of the Chain Distribution in the Proximal Range. Let us first consider the nonadsorbed chain in the presence of weak potential $u_{\text {ads }} \rightarrow 0$. Assuming a selfsimilar distribution, $\psi_{0}(x, \Theta)=x^{\alpha} g(\kappa)$ where $\kappa=\Theta\left(2 l_{\mathrm{p}} / x\right)^{1 / 3}$, we rewrite eq 6 with $u_{\text {ads }}=\epsilon_{0}=0$ as an eigenvalue problem for $g(\kappa)$

$$
-\frac{1}{\kappa} \frac{\partial^{2} g}{\partial \kappa^{2}}+\frac{\kappa}{3} \frac{\partial g}{\partial \kappa}=\alpha g
$$

Upon substitution $z=\kappa^{3 / 9}$, eq $\mathrm{B} 1$ reduces to the following equation

$$
z \frac{\mathrm{d}^{2} g}{\mathrm{~d} z^{2}}+\left(\frac{2}{3}-z\right) \frac{\mathrm{d} g}{\mathrm{~d} z}+\alpha g=0
$$

Under condition $g(z \rightarrow \infty) \rightarrow 0$, the only solution to eq B2 is $g(z) \approx \Psi(-\alpha, 2 / 3, z)$ where $\Psi(\chi, \omega, z)$ and $\Gamma(\chi)$ are defined in the main text. $\psi(x \rightarrow 0, \Theta<0) \rightarrow 0$ defines the spectrum of eigenvalues $\alpha_{n}=1 / 6-n$, where $n=0, \pm 1, \pm 2, \ldots$ (see Appendix $\mathrm{B}$ in ref 28). The requirement that $\psi$ does not have knots is satisfied for $n=0(\alpha=1 / 6)$ and $n=1(\alpha=-5 / 6)$, and the general solution for $u_{\mathrm{ads}}=0$ in the region $x>b$ is given by eq 7 of the main text.

To solve eq 8 , we substitute $C_{0}$ from the second equation into the first equation and multiply by $x^{2 / 3}$. We obtain

$$
x^{4 / 3} \frac{\mathrm{d}^{2} C_{1}}{\mathrm{~d} x^{2}}-\frac{2}{3} x^{1 / 3} \frac{\mathrm{d} C_{1}}{\mathrm{~d} x}+\left(\bar{F}_{11}+D x^{2 / 3}\right) C_{1}=0
$$


where $D=\bar{F}_{00} \bar{F}_{11}-\bar{F}_{01} \bar{F}_{10}$ and $\bar{F}_{n m}=F_{n m} \phi\left(2 l_{\mathrm{p}}\right)^{1 / 3}, n, m=0$, 1. Substituting $y=x^{1 / 3}$ into eq B3 and multiplying it by $y^{2}$, we get

$$
y^{2} \frac{\mathrm{d}^{2} C_{1}}{\mathrm{~d} y^{2}}-2 y \frac{\mathrm{d} C_{1}}{\mathrm{~d} y}+y^{2}\left(9 \bar{F}_{11}+9 D y^{2}\right) C_{1}=0
$$

Using $z=y^{2}$ allows us to rewrite eq B4 as

$$
\gamma_{2} z \frac{\mathrm{d}^{2} C_{1}}{\mathrm{~d} z^{2}}+\beta_{1} \frac{\mathrm{d} C_{1}}{\mathrm{~d} z}+\left(\gamma_{0} z+\beta_{0}\right) C_{1}=0
$$

where $\gamma_{0}=9 D, \beta_{0}=9 \bar{F}_{11}, \beta_{1}=-4$, and $\gamma_{2}=4$. The general solution of eq $\mathrm{B} 5$ is given by

$$
\begin{aligned}
C_{1}(x) & =\mathrm{e}^{3 / 2 \sqrt{-D} x^{2 / 3}}\left[c_{1} \Phi\left(\rho,-\frac{1}{2},-3 \sqrt{-D} x^{2 / 3}\right)\right. \\
& \left.+c_{2}\left(-3 \sqrt{-D} x^{2 / 3}\right)^{3 / 2} \Phi\left(\rho+\frac{3}{2}, \frac{5}{2},-3 \sqrt{-D} x^{2 / 3}\right)\right]
\end{aligned}
$$

where $c_{1}$ and $c_{2}$ are constants and $\rho=\left(3 \bar{F}_{11}-2 \sqrt{-D}\right) /$ $4 \sqrt{-D}$. In eq $\mathrm{B} 6, \Phi(k, l, x)$ is the Kummer series defined in the text. In the range $0 \leq x \leq b, \psi_{1}(x, \Theta=0)$ diverges as $x \rightarrow$ 0 . To avoid this divergence, we require that $C_{1}(x=0)=0$. This is satisfied when $c_{1}=0$. To ensure that $C_{1}(x) \rightarrow 0$ as $x \rightarrow$ $\infty$ for $x>b$, we set $c_{2}=0$. Substituting $\phi=\phi_{\text {in }}$ and $\phi=\phi_{\text {out }}$ into solutions for $0 \leq x \leq b$ and $x>b$ and using formulas $\Phi(k, l, x)=\mathrm{e}^{x} \Phi(l-k, l, x),\left(\mathrm{d}^{m}\right) /\left(\mathrm{d} x^{m}\right) \Phi(k, l, x)=\left((k)^{m}\right) /((l)-$ $m) \Phi(k+m, l+m, x)$ we obtain eq 9 .

\section{References and Notes}

(1) (a) Liphardt, G.; Smith, D.; Bustamante, C. Curr. Opin. Struct. Biol. 2000, 10, 279; (b) Allemand, J.-F; Bensimon, D.; Croquette, V. Curr. Opin. Struct. Biol. 2003, 13, 266.

(2) (a) Yin, H.; Wang, M. D.; Svoboda, K.; Landick, R.; Block, S. M.; Gelles, J. Science 1995, 270, 1653. (b) Bianco, P. R.; Brewer, L. R.; Corzett, M.; Balhorn, R.; Yeh, Y.; Kowalczykowski, S. C.; Baskin, R. J. Nature 2001, 409, 374.

(3) Alberts, B. Molecular Biology of the Cell, 1st ed.; Garland Publishing: New York, 1994.

(4) (a) Labeit, S.; Kolmerer, B. Science 1995, 270, 293. (b) Minajeva, A.; Kulke, M.; Fernandez, J. M.; Linke, W. A. Biophys. J. 2001, 80, 1442. (5) (a) Henrickson, S. E.; Misakian, M.; Robertson, B.; Kasianowicz, J. J. Phys. Rev. Lett. 2000, 85, 3057. (b) Kasianowicz, J. J.; Brandin, E.; Branton, D.; Deamer, D. W. Proc. Natl. Acad. Sci. U.S.A. 1996, 93, 13770.

(6) (a) Sung, W.; Park, P. J. Phys. Rev. Lett. 1996, 77, 783. (b) Muthukumar, M. J. Chem. Phys. 2003, 118, 5174; 1999, 111, 10371.

(7) (a) Marshall, B. T.; Long, M.; Piper, J. W.; Yago, T.; McEever, R. P.; Zhu, C. Nature 2003, 423, 190. (b) Ramachandran, V.; Yago, T.; Epperson, T. K.; Kobzdej, M. M.; Nollert, M. U.; Cummings, R. D.; Zhu, C.; McEver, R. P. Proc. Natl. Acad. Sci. U.S.A. 2001, 98, 10166.
(8) (a) Leppaenen, A.; White, S. P.; Helin, J.; McEver, R. P.; Cummings, R. D. J. Biol. Chem. 2000, 275, 39569. (b) Somers, W. S.; Tang, J.; Shaw, G. D.; Camphausen, R. T. Cell 2000, 103, 467.

(9) (a) de Gennes, P.-J. Scaling Concepts in Polymer Physics, 1st ed.; Cornell University Press: Ithaca, NY, 1979. (b) Freer, G.; Cohen-Stuart, M.; Scheutjens, J.; Cosgrove, T.; Vincent, B. Polymers at Interfaces, 1st ed.; Chapman and Hall: London, 1993.

(10) (a) Muthukumar, M. J. Chem. Phys. 1987, 86, 7230. (b) Muthukumar, M. J. Chem. Phys. 2004, 120, 9343. (c) Wiegel, F. W. J. Phys. A: Math. Gen. 1977, 10, 299.

(11) (a) Ellis, M.; Kong, C. Y.; Muthukumar, M. J. Chem. Phys. 2000, 112, 8723. (b) McNamara, J.; Kong, C. Y.; Muthukumar, M. J. Chem. Phys. 2002, 117, 5354 .

(12) (a) Semenov, A. N.; Joanny, J. F. Europhys. Lett. 1995, 29, 279. (b) Semenov, A. N.; Bonet-Avalos, J.; Johner, A.; Joanny, J. F. Macromolecules 1996, 29, 2179.

(13) (a) Weisel, J. W.; Shuman, H.; Litvinov, R. I. Curr. Opin. Struct. Biol. 2003, 13, 227. (b) Liphardt, J.; Dumont, S.; Smith, S. B.; Tinoco, I.; Bustamante, C. Science 2002, 296, 1832.

(14) (a) Chang, K. C.; Tees, D. F.; Hammer, D. A. Proc. Natl. Acad. Sci. U.S.A. 2000, 97, 11262. (b) Bustanji, Y.; Arciola, R. C.; Conti, M.; Mandello, E.; Montanaro, L.; Samori, B. Proc. Natl. Acad. Sci. U.S.A. 2003 $100,13292$.

(15) (a) Rief, M.; Clausen-Shaunman, H.; Gaub, H. E. Nat. Struct. Biol. 1999, 6, 346. (b) Rief, M.; Gautel, M.; Schemmel, A.; Gaub, H. E. Biophys. J. 1998, 75, 3008 .

(16) (a) Ha, T.; Zhuang, X.; Kim, H. D.; Orr, J. W.; Williamson, J. R.; Chu, S. Proc. Natl. Acad. Sci. U.S.A. 1999, 96, 9077. (b) Zhuang, X.; Bartley, L. E.; Babcock, H. P.; Russell, R.; Ha, T.; Herschlag, D.; Chu, S. Science 2000, 288, 2048

(17) Hyeon, C.; Thirumalai, D. Proc. Natl. Acad. Sci. U.S.A. 2003, 100 , 10249 .

(18) (a) Barsegov, V.; Thirumalai, D. Proc. Natl. Acad. Sci. U.S.A. 2005, 102, 1835. (b) Barsegov, V.; Thirumalai, D. Phys. Rev. Lett. 2005, 95, 168302 .

(19) (a) Bustamante, C.; Marko, J. F.; Siggia, E. D.; Smith, S. Science 1994, 265, 1599. (b) Smith, D. E.; Chu, S. Science 1998, 281, 1335. (c) Perkins, Th. T.; Smith, D. E.; Chu, S. Science 1997, 276, 2016.

(20) (a) Schurr, F.; MacKintosh, F. C.; Williams, D. R. M. Europhys. Lett. 2000, 51, 279. (b) Montesi, A.; Pasquali, M.; MacKintosh, F. C. Phys Rev. E 2004, 69, 021916.

(21) (a) Bouchiat, C.; Mezard, M. Phys. Rev. Lett. 1998, 80, 1556. (b) Marko, J.; Siggia, E. D. Macromolecules 1995, 28, 8759. (c) Wilhelm, J.; Frey, E. Phys. Rev. Lett. 1996, 77, 2581.

(22) (a) Samuel, J.; Sinha, S. Phys. Rev. E 2002, 66, 050801. (b) Krishna, M. M. G.; Samuel, J.; Sinha, S. J. Phys. A 2000, 33, 5965.

(23) (a) Lifshitz, I. M. Zh. Eksp. Teor. Fiz. 1968, 55, 2408. (b) Grosberg, A. Yu. Biofizika 1979, 24, 32 (Biophysics 1979, 24, 30).

(24) Kierfeld, J.; Lipowsky, R. J. Phys. A: Math. Gen. 2005, 38, L155.

(25) (a) Post, C. B.; Zimm, B. H. Biopolymers 1979, 18, 1487. (b) Bloomfield, V. A. Biopolymers 1997, 44, 269.

(26) (a) Noguchi, H.; Yoshikawa, K. J. Chem. Phys. 2000, 113, 854. (b) Sakaue, T.; Yoshikawa, K. J. Chem. Phys. 2002, 117, 6323.

(27) (a) Kuznetsov, D. B.; Sung, W. J. Chem. Phys. 1997, 107, 4729. (b) Macromolecules 1998, 31, 2679.

(28) Semenov, A. N. Eur. Phys. J. E 2002, 9, 353.

(29) Abramowitz, M.; Stegun, I. Handbook of Mathematical Functions, 9th ed.; Dover: New York, 1972.

(30) Caliskan, G. Phys. Rev. Lett., submitted for publication.

(31) Schuler, B.; Lipman, E. A.; Steinbach, P. J.; Kumke, M.; Eaton, W. A. Proc. Natl. Acad. Sci. U.S.A. 2005, 102, 2754.

(32) Murayama, Y.; Sakamaki, Y.; Sano, M. Phys. Rev. Lett. 2003, 90, 018102

(33) Lee, N.; Thirumalai, D. Eur. Phys. J. B 1999, 12, 599. 